\title{
1 Supplementary tables
}

The new calibration of climate reconstruction for Mongolia and Siberia presented in this study is based up on the New Mongolia-Siberia Data Base (NMSDB). Published br-GDGT/climate calibration equations are tested on the (NMSDB) (S1). Location, ecosystems as well as sample type are provided in Table (S2). All the br-GDGTs mr-models discussed in this study 5 are presented in the table (S3). 


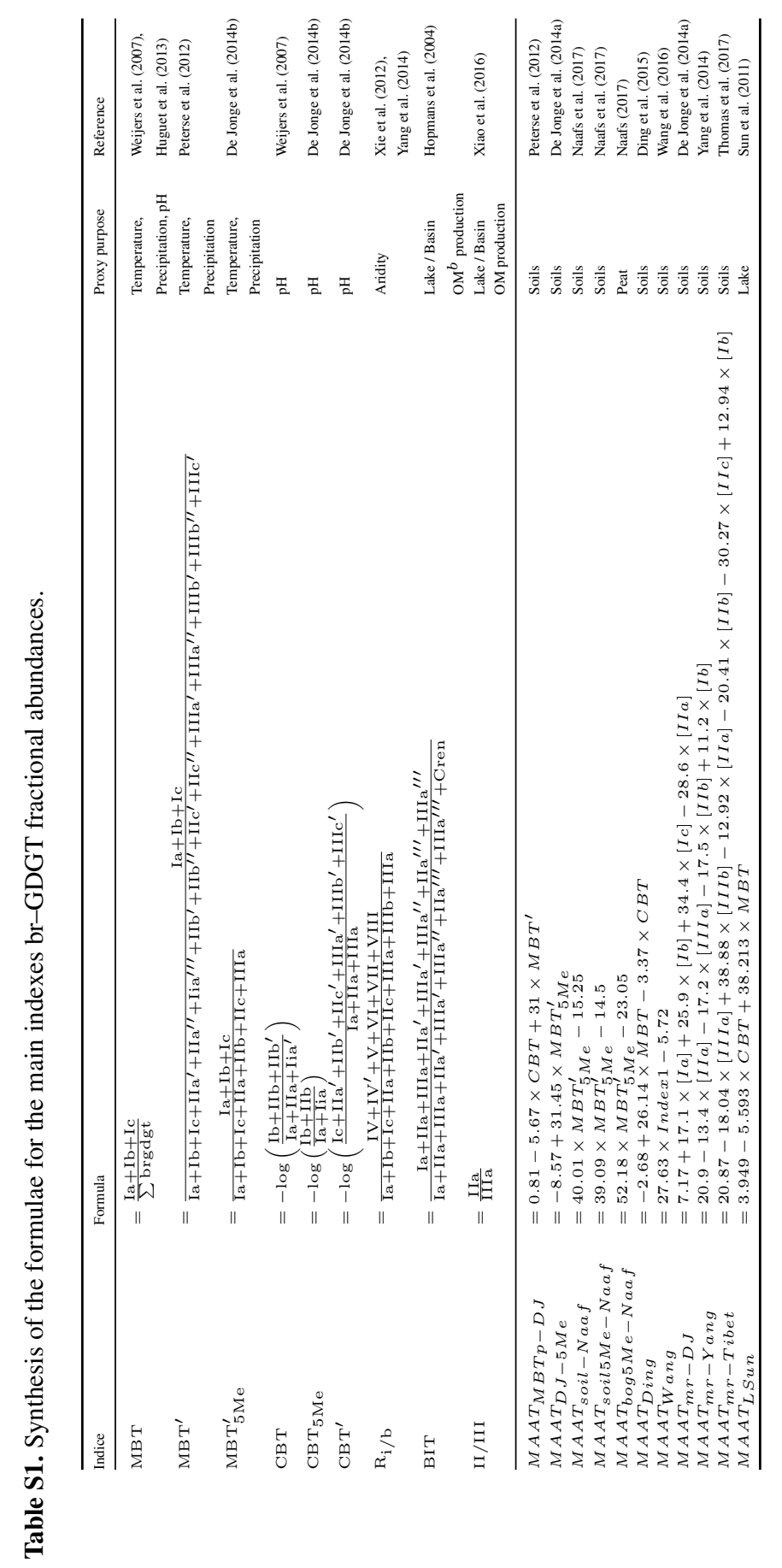


Table S2. Sample sites included in the New Mongolia-Siberia DataBase (NMSDB).

\begin{tabular}{|c|c|c|c|c|c|c|c|}
\hline Site Label & Lat. & Long. & Elev. & Pollen & GDGT & Type used in Fig. & Biomes \\
\hline MMNT1M01 & 48.3983 & 106.8594 & 1137 & Moss polster & Soil part of moss litter & Moss & Steppe-forest \\
\hline MMNT1M02 & 48.4014 & 106.8613 & 1161 & Moss polster & Soil part of moss litter & Moss & Steppe-forest \\
\hline MMNT2M01 & 48.4472 & 107.0542 & 1438 & Moss polster & Soil part of moss litter & Moss & Steppe-forest \\
\hline MMNT2M02 & 48.4460 & 107.0551 & 1333 & Moss polster & Soil part of moss litter & Moss & Steppe-forest \\
\hline MMNT2M03 & 48.4449 & 107.0564 & 1265 & Moss polster & Soil part of moss litter & Moss & Steppe-forest \\
\hline MMNT2M04 & 48.4441 & 107.0580 & 1266 & Moss polster & Soil part of moss litter & Moss & Light taiga \\
\hline MMNT2M05 & 48.4425 & 107.0593 & 1262 & Moss polster & Unused & Moss & Light taiga \\
\hline MMNT2M05, & 48.4444 & 107.0634 & 1273 & Moss polster & Soil part of moss litter & Moss & Light taiga \\
\hline MMNT2M06 & 48.4412 & 107.0629 & 1328 & Moss polster & Soil part of moss litter & Moss & Light taiga \\
\hline MMNT2M07 & 48.4381 & 107.0660 & 1475 & Moss polster & Soil part of moss litter & Moss & Steppe-forest \\
\hline MMNT3S01 & 47.2993 & 103.6092 & 1323 & Soil & Soil & Soil & Steppe \\
\hline MMNT3S02 & 47.2000 & 102.8438 & 1457 & Soil & Soil & Soil & Steppe \\
\hline MMNT3M03 & 46.8239 & 102.2307 & 1669 & Moss polster & Soil part of moss litter & Moss & Alpine meadow \\
\hline MMNT3M04 & 46.7932 & 102.0868 & 1734 & Moss polster & Soil part of moss litter & Moss & Alpine meadow \\
\hline MMNT3S05 & 46.7800 & 101.9510 & 1830 & Soil & Soil & Soil & Alpine meadow \\
\hline MMNT4S01 & 45.6645 & 101.6054 & 1750 & Soil & Soil & Soil & Steppe-desert \\
\hline MMNT4S02 & 45.1759 & 101.4288 & 1238 & Soil & Soil & Soil & Desert \\
\hline MMNT4S03 & 45.1724 & 101.4517 & 1233 & Soil & Soil & Soil & Desert \\
\hline MMNT4S04 & 45.1702 & 101.4806 & 1230 & Soil & Soil & Soil & Desert \\
\hline MMNT4S05 & 45.1618 & 101.4927 & 1228 & Soil & Soil & Soil & Desert \\
\hline MMNT4S06 & 45.1467 & 101.5083 & 1230 & Soil & Soil & Soil & Desert \\
\hline MMNT4S07 & 45.1402 & 101.5087 & 1232 & Soil & Soil & Soil & Desert \\
\hline MMNT4S08 & 44.6746 & 102.1844 & 1508 & Soil & Soil & Soil & Steppe-desert \\
\hline MMNT4M09 & 44.4509 & 102.3459 & 1847 & Moss polster & Soil part of moss litter & Moss & Steppe-desert \\
\hline MMNT4M10 & 44.3952 & 102.4511 & 1677 & Moss polster & Soil part of moss litter & Moss & Steppe-desert \\
\hline MMNT4S11 & 44.1685 & 102.6031 & 1273 & Soil & Soil & Soil & Desert \\
\hline MMNT4S12 & 43.9494 & 102.7411 & 1574 & Soil & Soil & Soil & Steppe-desert \\
\hline MMNT4S13 & 43.8636 & 102.7479 & 1802 & Unused & Soil & Soil & Steppe-desert \\
\hline MMNT4S14 & 43.7650 & 102.8018 & 1982 & Soil & Soil & Soil & Steppe-desert \\
\hline MMNT5C01 & 48.4074 & 101.8797 & 1433 & Pond mud & Pond mud & Mud & Alpine meadow \\
\hline MMNT5C03 & 48.6592 & 101.2015 & 1579 & Pond mud & Pond mud & Mud & Alpine meadow \\
\hline MMNT5M04 & 48.4136 & 102.2389 & 1566 & Moss polster & Soil part of moss litter & Moss & Steppe \\
\hline MMNT5M05 & 48.4203 & 102.2266 & 1538 & Moss polster & Soil part of moss litter & Moss & Steppe \\
\hline MMNT5M06 & 47.7340 & 101.2459 & 1646 & Moss polster & Unused & Moss & Steppe-forest \\
\hline MMNT5M07 & 47.7338 & 101.2460 & 1647 & Moss polster & Unused & Moss & Steppe-forest \\
\hline MMNT5C11 & 48.9290 & 101.9588 & 1316 & Lake Top-core & Unused & Lake sediment & Steppe-forest \\
\hline MMNT5C12 & 48.6907 & 101.4263 & 1436 & Lake Top-core & Cross-value & Lake sediment & Steppe-forest \\
\hline MRUT1M01 & 52.0497 & 104.1132 & 565 & Moss polster & Soil part of moss litter & Moss & Dark taiga \\
\hline MRUT1M02 & 52.0498 & 104.1137 & 574 & Moss polster & Soil part of moss litter & Moss & Dark taiga \\
\hline MRUT1M03 & 52.0493 & 104.1132 & 582 & Moss polster & Soil part of moss litter & Moss & Light taiga \\
\hline MRUT1M04 & 52.0500 & 104.1140 & 557 & Moss polster & Soil part of moss litter & Moss & Dark taiga \\
\hline MRUT1M05 & 52.0328 & 104.2263 & 640 & Moss polster & Soil part of moss litter & Moss & Light taiga \\
\hline MRUT1M06 & 52.0148 & 104.2612 & 554 & Moss polster & Soil part of moss litter & Moss & Dark taiga \\
\hline MRUT1M07 & 52.0046 & 104.3738 & 476 & Moss polster & Soil part of moss litter & Moss & Dark taiga \\
\hline MRUT1M08 & 51.9952 & 104.4023 & 471 & Moss polster & Soil part of moss litter & Moss & Light taiga \\
\hline MRUT1M09 & 51.9900 & 104.4025 & 473 & Moss polster & Soil part of moss litter & Moss & Dark taiga \\
\hline MRUT1M10 & 51.9392 & 104.4636 & 538 & Moss polster & Soil part of moss litter & Moss & Light taiga \\
\hline MRUT1M11 & 51.9119 & 104.5331 & 725 & Moss polster & Soil part of moss litter & Moss & Light taiga \\
\hline MRUT1M12 & 51.8797 & 104.6266 & 772 & Moss polster & Soil part of moss litter & Moss & Light taiga \\
\hline Total & & & & 48 & 44 & 49 & \\
\hline
\end{tabular}


Table S3. Statistical values and equations of all the br-GDGT MAAT $m$ and $\mathrm{MAP}_{\mathrm{mr}}$ models.

\begin{tabular}{|c|c|c|c|c|c|}
\hline Model & $\mathrm{k}$ & $R^{2}$ & RMSE & AIC & Formula \\
\hline MAAT $_{\mathrm{mr} 1}$ & 1 & 0.38 & 1.5 & 168.4 & MAAT $_{\operatorname{mr} 1}=-0.5 \times 1+12.9 \times\left[\mathrm{IIIa}^{\prime}\right]$ \\
\hline MAAT $_{\mathrm{mr} 2}$ & 2 & 0.52 & 1.4 & 162.6 & MAAT $_{\mathrm{mr} 2}=-0.7 \times 1+13.4 \times\left[\mathrm{IIIa}^{\prime}\right]+11.8 \times[\mathrm{IIIb}]$ \\
\hline $\mathrm{MAAT}_{\mathrm{mr} 3}{ }^{a}$ & 3 & 0.57 & 1.3 & 156.4 & $\begin{array}{l}\text { MAAT }_{\operatorname{mr} 3}=0.6 \times 1-25.1 \times[\mathrm{IIIa}]+12.3 \\
\times\left[\mathrm{IIIa}^{\prime}\right]+7.2 \times[\mathrm{Ib}]\end{array}$ \\
\hline MAAT $_{\mathrm{mr} 4}$ & 5 & 0.62 & 1.2 & 152.6 & $\begin{array}{l}\text { MAAT } \operatorname{mr} 4=4.5 \times 1-36.8 \times[\mathrm{IIIa}] \\
+7.3 \times\left[\mathrm{IIIa}^{\prime}\right]-37.2 \times[\mathrm{III}]-24 \times[\mathrm{II}]-5.2 \times[\mathrm{Ia}]\end{array}$ \\
\hline MAAT $_{\mathrm{mr} 5}{ }^{b}$ & 7 & 0.66 & 1.1 & 153.9 & $\begin{array}{l}\operatorname{MAAT}_{\operatorname{mr} 5}=4.8 \times 1-38.5 \times[\mathrm{III}]+7.9 \times\left[\mathrm{IIIa}^{\prime}\right] \\
-27.3 \times[\mathrm{III}]-3.3 \times\left[\mathrm{IIa}^{\prime}\right]-26.3 \times[\mathrm{IIb}]+8.5 \times\left[\mathrm{IIb}^{\prime}\right] \\
-5.6 \times[\mathrm{Ia}]\end{array}$ \\
\hline MAAT $_{\mathrm{mr} 6}$ & 9 & 0.67 & 1.1 & 155.7 & $\begin{array}{l}\text { MAAT }_{\operatorname{mr} 6}=12.3 \times 1-52.1 \times[\mathrm{IIIa}]-16.9 \times[\mathrm{IIIb}] \\
-25.9 \times\left[\mathrm{IIIb}^{\prime}\right]-41.1 \times[\mathrm{III}]-6 \times[\mathrm{IIa}]-10.4 \times\left[\mathrm{IIa}^{\prime}\right] \\
-38.5 \times[\mathrm{IIb}]-13.3 \times[\mathrm{Ia}]-32.8 \times[\mathrm{Ic}]\end{array}$ \\
\hline MAAT $_{\mathrm{mr} 7}$ & 10 & 0.68 & 1.1 & 157.2 & $\begin{array}{l}\text { MAAT }_{\mathrm{mr} 7}=12.3 \times 1-52.6 \times[\mathrm{IIIa}]-16.8 \times[\mathrm{III} \mathrm{Ib}] \\
-25.3 \times\left[\mathrm{IIIb}^{\prime}\right]-35.7 \times[\mathrm{IIIc}]-6 \times[\mathrm{IIa}]-10.5 \times\left[\mathrm{IIa}^{\prime}\right] \\
-37.8 \times[\mathrm{II}]-15.4 \times[\mathrm{II} c]-13.2 \times[\mathrm{Ia}]-31.4 \times[\mathrm{Ic}]\end{array}$ \\
\hline MAAT $_{\mathrm{mr} 8}$ & 12 & 0.69 & 1.1 & 160.5 & $\begin{array}{l}\text { MAAT }_{\mathrm{mr} 8}=12.5 \times 1-54.9 \times[\mathrm{IIIa}]-23.6 \times[\mathrm{IIIb}] \\
-26.8 \times\left[\mathrm{IIIb}^{\prime}\right]-35.1 \times[\mathrm{IIIc}]-23.4 \times\left[\mathrm{IIIc}^{\prime}\right]-5.9 \times[\mathrm{IIa}] \\
-10.4 \times\left[\mathrm{IIa}^{\prime}\right]-40.6 \times[\mathrm{IIb}]-16 \times[\mathrm{IIc}]-13.5 \times[\mathrm{Ia}] \\
+5.6 \times[\mathrm{Ib}]-35.8 \times[\mathrm{Ic}]\end{array}$ \\
\hline MAAT $_{\text {mr9 }}$ & 15 & 0.69 & 1.1 & 166.2 & $\begin{array}{l}\text { MAAT }_{\mathrm{mr} 9}=10.4 \times 1-49.5 \times[\mathrm{IIIa}]+3.3 \times\left[\mathrm{IIIa}^{\prime}\right] \\
-21.5 \times[\mathrm{IIIb}]-26.2 \times\left[\mathrm{IIIb}^{\prime}\right]-30.9 \times[\mathrm{IIIc}]-23.3 \times\left[\mathrm{IIIc}^{\prime}\right] \\
-4.4 \times[\mathrm{IIa}]-8.9 \times\left[\mathrm{IIa}^{\prime}\right]-38.1 \times[\mathrm{IIb}]+0.3 \times\left[\mathrm{IIb}^{\prime}\right] \\
-13.2 \times[\mathrm{IIc}]+0 \times\left[\mathrm{IIc}^{\prime}\right]-11.1 \times[\mathrm{Ia}]+7.7 \times[\mathrm{Ib}]-31.7 \times[\mathrm{Ic}]\end{array}$ \\
\hline $\mathrm{MAP}_{\mathrm{mr} 1}$ & 1 & 0.34 & 112 & 546.1 & $\mathrm{MAP}_{\mathrm{mr} 1}=179.3 \times 1+509.1 \times[\mathrm{Ia}]$ \\
\hline $\mathrm{MAP}_{\mathrm{mr} 2}$ & 2 & 0.51 & 96.1 & 534.6 & $\mathrm{MAP}_{\mathrm{mr} 2}=59.6 \times 1+2289.9 \times\left[\mathrm{IIb}^{\prime}\right]+710.8 \times[\mathrm{Ia}]$ \\
\hline $\mathrm{MAP}_{\mathrm{mr} 3}$ & 3 & 0.58 & 91.3 & 532.1 & $\begin{array}{l}\mathrm{MAP}_{\mathrm{mr} 3}=94.1 \times 1+2319.7 \times\left[\mathrm{IIb}^{\prime}\right]+702.2 \times[\mathrm{Ia}] \\
-3752.5 \times[\mathrm{Ic}]\end{array}$ \\
\hline $\mathrm{MAP}_{\mathrm{mr} 4}$ & 5 & 0.65 & 82 & 526.7 & $\begin{array}{l}\mathrm{MAP}_{\mathrm{mr} 4}=245.1 \times 1-666.7 \times\left[\mathrm{IIIa}^{\prime}\right]+2431.9 \times\left[\mathrm{IIb}^{\prime}\right] \\
+396.2 \times[\mathrm{Ia}]-276 \times[\mathrm{Ib}]-3780 \times[\mathrm{Ic}]\end{array}$ \\
\hline $\mathrm{MAP}_{\mathrm{mr} 5}$ & 8 & 0.69 & 75.9 & 525.8 & $\begin{array}{l}\mathrm{MAP}_{\operatorname{mr} 5}=-103.8 \times 1+1553.4 \times[\mathrm{III}]+536 \times[\mathrm{III} \mathrm{Ib}] \\
-3145.6 \times\left[\mathrm{IIIb}^{\prime}\right]+2480.3 \times[\mathrm{III}]+1290.1 \times[\mathrm{II} b] \\
+2459.3 \times\left[\mathrm{IIb}^{\prime}\right]+927.9 \times[\mathrm{Ia}]-2955.3 \times[\mathrm{Ic}]\end{array}$ \\
\hline $\mathrm{MAP}_{\mathrm{mr} 6}{ }^{a}$ & 10 & 0.73 & 72.5 & 525.8 & $\begin{array}{l}\text { MAP } \mathrm{mr} 6=-511.3 \times 1+1205.9 \times[\mathrm{IIIa}]+1387.2 \times[\mathrm{IIIb}] \\
+738.8 \times\left[\mathrm{IIa}^{2}+969.8 \times\left[\mathrm{IIa}^{\prime}\right]+1957.1 \times[\mathrm{IIb}]\right. \\
+3006.3 \times\left[\mathrm{IIb}^{\prime}\right]+2406.4 \times[\mathrm{IIC}]+2003.1 \times\left[\mathrm{IIc}^{\prime}\right] \\
+1081.7 \times\left[\mathrm{Ia}^{\prime}\right]-2406.3 \times[\mathrm{Ic}]\end{array}$ \\
\hline $\mathrm{MAP}_{\mathrm{mr} 7}^{b}$ & 12 & 0.75 & 68.5 & 524.8 & $\begin{array}{l}\text { MAP }_{\operatorname{mr} 7}=-502.6 \times 1+1359.5 \times[\mathrm{IIIa}]+2462.5 \times[\mathrm{IIIb}] \\
-2178.3 \times\left[\mathrm{IIIb}^{\prime}\right]+657.7 \times\left[\mathrm{IIa}^{\prime}+986.8 \times\left[\mathrm{IIa}^{\prime}\right]\right. \\
+2440.5 \times[\mathrm{IIb}]+3423.5 \times\left[\mathrm{IIb}^{\prime}\right]+2831.2 \times[\mathrm{IIc}] \\
+1967.2 \times\left[\mathrm{IIc}^{\prime}\right]+1150.2 \times[\mathrm{Ia}]-955.6 \times[\mathrm{Ib}]-2103.4 \times[\mathrm{Ic}]\end{array}$ \\
\hline $\mathrm{MAP}_{\mathrm{mr} 8}$ & 15 & 0.76 & 67.9 & 530 & $\begin{array}{l}\mathrm{MAP}_{\mathrm{mr} 8}=-619.5 \times 1+1725.6 \times[\mathrm{III}]+161.7 \times\left[\mathrm{IIII}^{\prime}\right] \\
+2603.5 \times[\mathrm{III}]-2069.7 \times\left[\mathrm{III}^{\prime}\right]+380.3 \times[\mathrm{III} \mathrm{c}] \\
+2226.9 \times\left[\mathrm{IIIc}^{\prime}\right]+730.5 \times\left[\mathrm{IIa}^{\prime}\right]+1028.2 \times\left[\mathrm{IIa}^{\prime}\right] \\
+2569 \times[\mathrm{II} b]+3424.2 \times\left[\mathrm{IIb}^{\prime}\right]+2734.6 \times[\mathrm{IIc}]+1830.5 \times\left[\mathrm{IIc}^{\prime}\right] \\
+1289.3 \times[\mathrm{Ia}]-854.1 \times[\mathrm{Ib}]-1745.6 \times[\mathrm{Ic}]\end{array}$ \\
\hline
\end{tabular}

${ }^{a}$ : The models displayed in blue have the best Siberian-Mongolian data cross-fit. ${ }^{b}:$ The one in red are the most universal models. 


\section{Supplementary figures}

To help the understanding of the limits and uncertainties of our calibrations, the correlation between the climate parameters from the WorldClim 2 modern climate modelling are presented in the figure S1. Then the figure S2 present in three parcels the NMSDB br-GDGTs data set in comparison with the values from the world br-GDGT data bases. Finally, some of the brGDGTs-climate models designed in this study are presented in figure S3 : the mr-models as well as the MBT and CBT models.

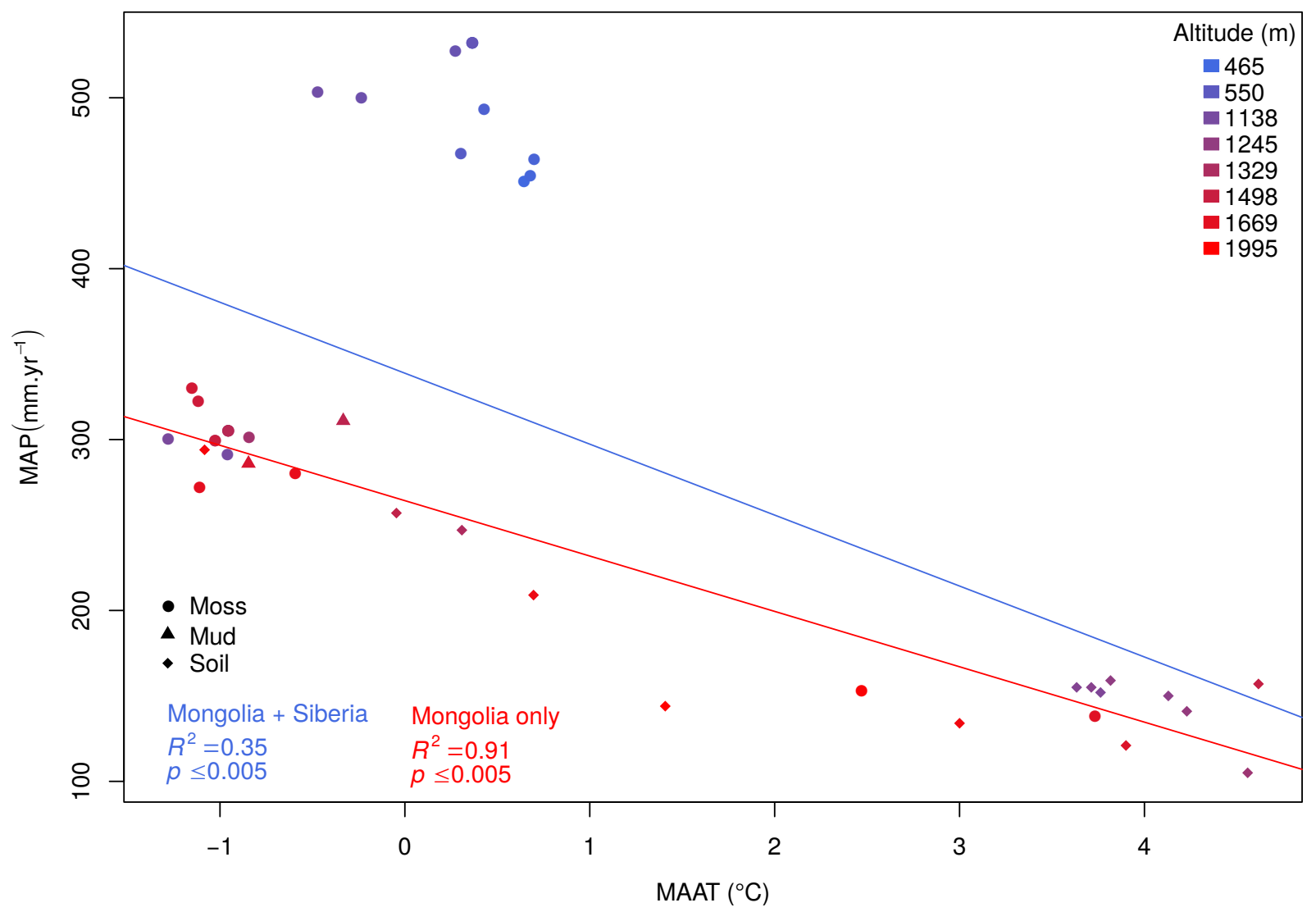

Figure S1. Relation between MAAT and MAP for all the sites of the NMSDB extracted from WorldClim2 (Fick and Hijmans, 2017). Shapes illustrate the type of sample (dot for moss, triangle for mud and lozenge for soil samples) and the red-blue coloured range highlight the altitude gradient. The two curves display the linear regression of the MAP-MAAT correlation in the full NMSDB (blue) and the Mongolian subplot (red). 

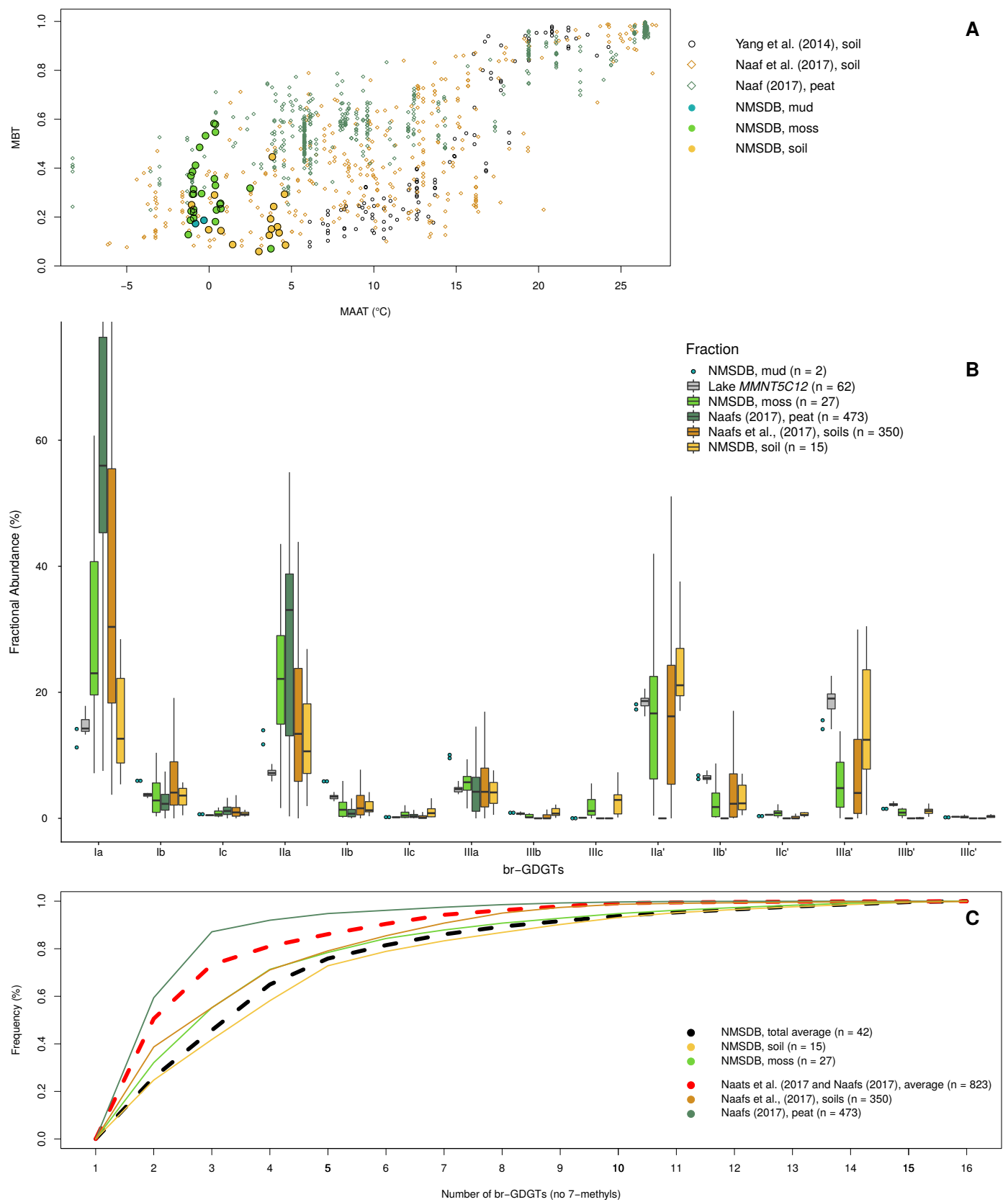

Figure S2. Global comparison between the NMSDB and some world surface brGDGT results. Comparison of the dispersion of the NMSDB samples in front of the global databases for (A) the MBT-MAAT relation. B: Histogram of the brGDGT fractional abundance. C: Cumulative curve of the br-GDGT average values. Global databases from Yang et al. (2014); Naafs et al. (2017); Naafs (2017). 

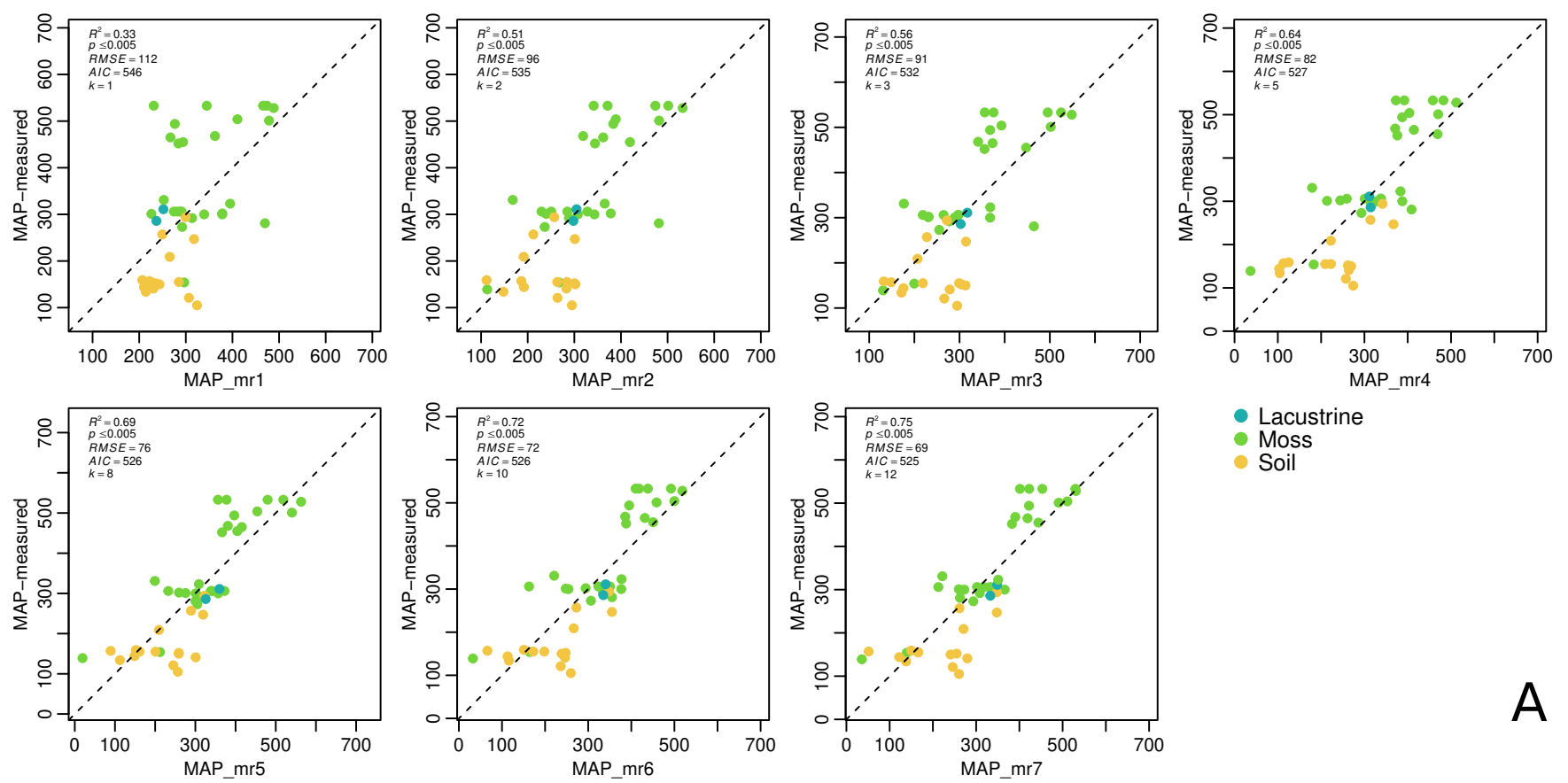

- Lacustrine

- Moss

Soil
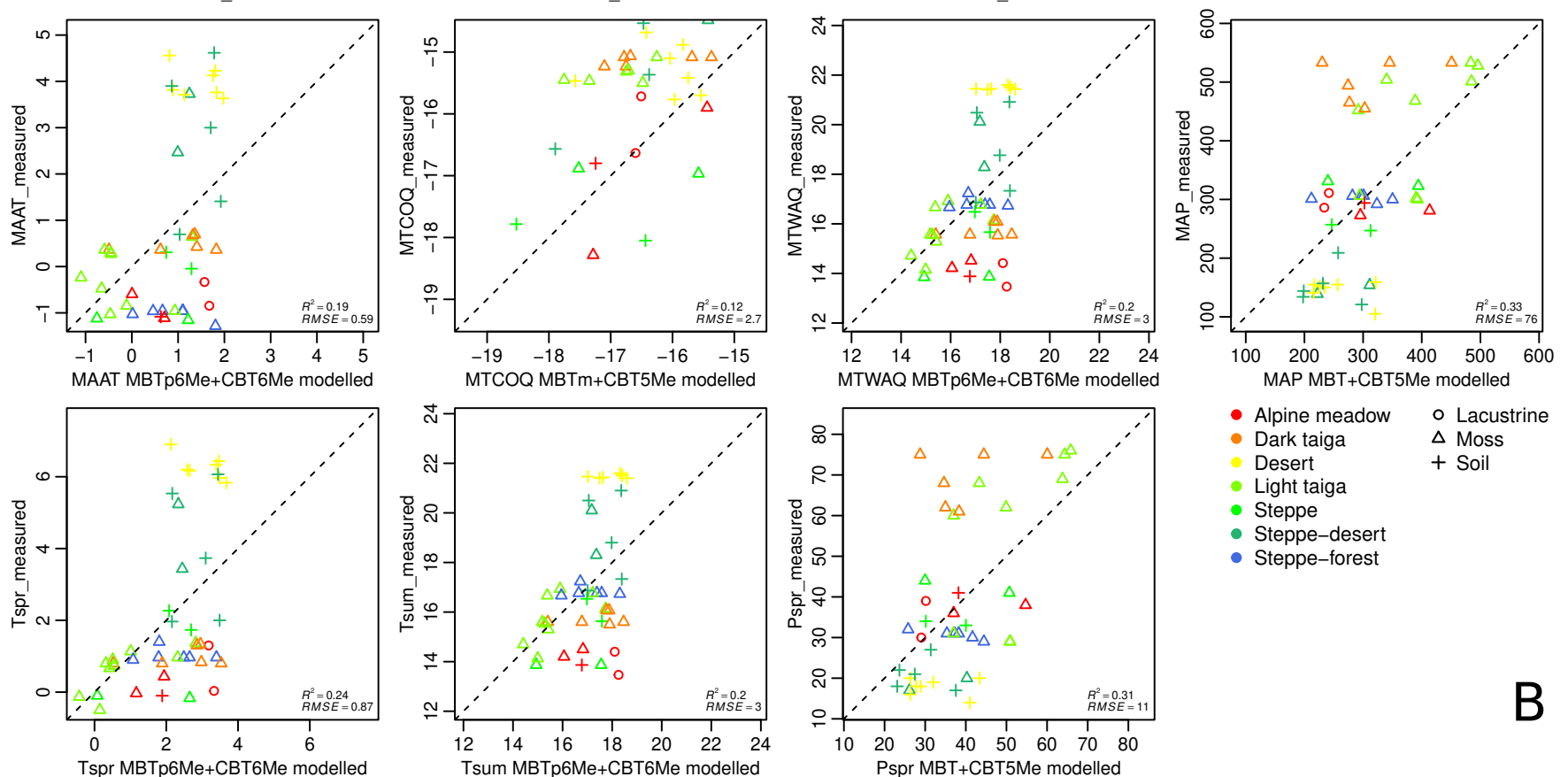

MAP MBT+CBT5Me modelled

- Alpine meadow $\quad$ Lacustrine

- Dark taiga $\Delta$ Moss

Desert

+ Soil

Light taiga

- Steppe

- Steppe-desert

- Steppe-forest

A

Figure S3. A : Cross-plot for $\mathrm{MAP}_{\mathrm{mr}}$ model. The parcels are sorted by increasing parameter number. B : Cross-plot for selected climate MBT-CBT models. 


\section{References}

De Jonge, C., Hopmans, E. C., Zell, C. I., Kim, J.-H., Schouten, S., and Sinninghe Damsté, J. S.: Occurrence and abundance of 6-methyl branched glycerol dialkyl glycerol tetraethers in soils: Implications for palaeoclimate reconstruction, Geochimica et Cosmochimica Acta, 141, 97-112, 2014a.

De Jonge, C., Stadnitskaia, A., Hopmans, E. C., Cherkashov, G., Fedotov, A., and Sinninghe Damsté, J. S.: In situ produced branched glycerol dialkyl glycerol tetraethers in suspended particulate matter from the Yenisei River, Eastern Siberia, Geochimica et Cosmochimica Acta, 125, 476-491, 2014b.

Ding, S., Xu, Y., Wang, Y., He, Y., Hou, J., Chen, L., and He, J.-S.: Distribution of branched glycerol dialkyl glycerol tetraethers in surface soils of the Qinghai-Tibetan Plateau: implications of brGDGTs-based proxies in cold and dry regions, Biogeosciences, 12, 3141-3151, 2015.

Fick, S. E. and Hijmans, R. J.: WorldClim 2: new 1-km spatial resolution climate surfaces for global land areas: NEW CLIMATE SURFACES FOR GLOBAL LAND AREAS, International Journal of Climatology, 37, 4302-4315, 2017.

Hopmans, E. C., Weijers, J. W., Schefus s, E., Herfort, L., Damsté, J. S. S., and Schouten, S.: A novel proxy for terrestrial organic matter in sediments based on branched and isoprenoid tetraether lipids, Earth and Planetary Science Letters, 224, 107-116, 2004.

Huguet, A., Fosse, C., Laggoun-Défarge, F., Delarue, F., and Derenne, S.: Effects of a short-term experimental microclimate warming on the abundance and distribution of branched GDGTs in a French peatland, Geochimica et Cosmochimica Acta, 105, 294-315, 2013.

Naafs, B., Gallego-Sala, A., Inglis, G., and Pancost, R.: Refining the global branched glycerol dialkyl glycerol tetraether (brGDGT) soil temperature calibration, Organic Geochemistry, 106, 48-56, 2017.

30 Naafs, B. D. A.: Global biomarker (GDGT) database for peatlands, https://doi.org/https://doi.org/10.1594/PANGAEA.883765, https://doi. pangaea.de/10.1594/PANGAEA.883765, 2017.

Peterse, F., van der Meer, J., Schouten, S., Weijers, J. W., Fierer, N., Jackson, R. B., Kim, J.-H., and Damsté, J. S. S.: Revised calibration of the MBT-CBT paleotemperature proxy based on branched tetraether membrane lipids in surface soils, Geochimica et Cosmochimica Acta, 96, 215-229, 2012.

Sun, Q., Chu, G., Liu, M., Xie, M., Li, S., Ling, Y., Wang, X., Shi, L., Jia, G., and Lü, H.: Distributions and temperature dependence of branched glycerol dialkyl glycerol tetraethers in recent lacustrine sediments from China and Nepal, Journal of Geophysical Research: Biogeosciences, 116, 2011.

Thomas, E. K., Clemens, S. C., Sun, Y., Huang, Y., Prell, W., Chen, G., Liu, Z., and Loomis, S.: Midlatitude land surface temperature impacts the timing and structure of glacial maxima, Geophysical Research Letters, 44, 984-992, 2017.

40 Wang, H., Liu, W., and Lu, H.: Appraisal of branched glycerol dialkyl glycerol tetraether-based indices for North China, Organic Geochemistry, 98, 118-130, 2016.

Weijers, J. W., Schouten, S., van den Donker, J. C., Hopmans, E. C., and Damsté, J. S. S.: Environmental controls on bacterial tetraether membrane lipid distribution in soils, Geochimica et Cosmochimica Acta, 71, 703-713, 00503, 2007.

Xiao, W., Wang, Y., Zhou, S., Hu, L., Yang, H., and Xu, Y.: Ubiquitous production of branched glycerol dialkyl glycerol tetraethers (brGDGTs) in global marine environments: a new source indicator for brGDGTs, Biogeosciences, 13, 5883-5894, 2016.

Xie, S., Pancost, R. D., Chen, L., Evershed, R. P., Yang, H., Zhang, K., Huang, J., and Xu, Y.: Microbial lipid records of highly alkaline deposits and enhanced aridity associated with significant uplift of the Tibetan Plateau in the Late Miocene, Geology, 40, 291-294, 00080, 2012.

Yang, H., Pancost, R. D., Dang, X., Zhou, X., Evershed, R. P., Xiao, G., Tang, C., Gao, L., Guo, Z., and Xie, S.: Correlations between microbial tetraether lipids and environmental variables in Chinese soils: Optimizing the paleo-reconstructions in semi-arid and arid regions, Geochimica et Cosmochimica Acta, 126, 49-69, 2014. 